\title{
Purification of clathrin assembly protein from rat liver
}

\author{
Hyung-Lae Kim ${ }^{1,2}$ and Jeong-Ah Kim ${ }^{1}$ \\ ${ }^{1}$ Department of Biochemistry, Medical College, \\ Ewha Womans University, Seoul 158-710, Korea \\ ${ }^{2}$ Corresponding author: Tel, +82-2-650-5727; Fax, +82-2-650-5791; \\ E-mail: hyung@mm.ewha.ac.kr
}

Accepted 8 December 2000

Abbreviations: CCV, clathrin-coated vesicle; CALM, clathrin assembly protein lymphoid myeloid; FPLC, fast performance liquid chromatography; SDS-PAGE, sodium dodecylsulfate-polyacrylamide gel electrophoresis

\begin{abstract}
Recently, the gene encoding clathrin assembly protein of lymphoid myeloid leukemia (CALM), which is homologous to the AP180, was cloned from rat brain, and its expression differential to AP180 was reported (Kim and Lee, 1999). This gene product promotes the polymerization of clathrin into clathrin cage and found to be a regulator in membrane trafficking between intracellular compartments in eukaryotic cells (Kim et al., 2000). In this study, we have purified the CALM protein from clathrin-coated vesicles of rat liver using the monoclonal antibody against the recombinant $\mathrm{N}$-terminal region of the CALM. The coated proteins extracted from the coated vesicle fraction was further purified by multi-step procedures involving gel-filtration and ion-exchange chromatography and SDS-PAGE. The purified protein with an apparent molecular weight of $100 \mathrm{kD}$ promoted the assembly of clathrin triskelia into clathrin cage. In this respect the CALM protein bears a functional resemblance to the AP180 that has been previously described.
\end{abstract}

Keywords: clathrin-coated veside, CALM, AP180, assembly

\section{Introduction}

In eukaryotic cells, clathrin-coated vesicles (CCVs) are involved in membrane trafficking of ligands including receptor-mediated intracellular transport, transfer of proteins from trans-Golgi network to pre-lysosomal compartment and recycling of synaptic vesicles (Pearse and Robinson, 1990). The CCV coat is formed by polymeri- zation of triskelion-shaped clathrin molecules into lattice of clathrin cage, and is catalyzed by the assembly proteins. A major coating protein is clathrin, which consists of triskelion having three identical $190 \mathrm{kD}$ heavy chains and three 23-27 kD light chains. Coated vesicle also contains one or more assembly proteins (Robinson, 1994). They are adaptors and believed to link receptors to the clathrin network. The assembly protein promotes to assemble clathrin triskelia into artificial clathrin cage that resembles a coated vesicle. To date, six different adaptor proteins are known: AP-1, AP-2, AP-3, AP-4, AP180 and CALM. The AP-1, AP-2, AP-3, and AP-4 are tetramers, whereas AP180 and CALM are monomers.

AP180 is well studied because it is monomeric and neuron specific protein (Kondury and Roland, 1988; Zhou et al., 1992). AP180 was first discovered as a specific coating component of CCVs from neural tissue (Stephan et al., 1990). The native protein was shown to interact with clathrin triskelia and to induce clathrin assembly into a homogeneous population of $60-70 \mathrm{~nm}$ coats (Zhou et al., 1993). AP180 is phosphorylated at serine residues (Morris et al., 1990). The disassembly of clathrin from the vesicular coat was promoted by the phosphorylation of AP's (Wilde and Brodsky, 1996). Two primary functional properties of the AP180 are clathrin assembly and high affinity for specific inositol polyphosphates (Morris et al., 1993; Norris et al., 1995; Ye et al., 1995).

The CALM gene was cloned from human leukemia cell line, and was shown to be homologous to the monomeric clathrin adaptor protein of AP180 (Dreyling et al., 1996; Kim and Lee, 1999). In order to elucidate the role of CALM, we have expressed the full length of the CALM CDNA as GST-CALM fusion protein in bacterial system and reported the properties of the CALM protein, for the first time (Kim et al., 2000). The CALM protein expressed in bacteria has a molecular weight of $80 \mathrm{kD}$. The protein could promote clathrin triskelia into clathrin cages and could bind the preformed clathrin cage. However, N-terminal domain of CALM with a molecular weight of $33 \mathrm{kD}$ could not bind to preassembled clathrin cage, but assemble clathrin triskelia into clathrin cages. The CALM protein was bound to $\mathrm{SH} 3$ domain through $\mathrm{N}$-terminal domain, in vitro. The CALM protein is proteolyzed by caspase 3 , caspase 8 and calpain through C-terminal domain. Although molecular properties of the CALM were elucidated using the bacterial expression system, native forms of CALM in eukaryotes and its characteristics were not known.

In this study, to understand the role of CALM protein in mammalian cells, we have purified the CALM protein from rat liver, through various procedures including CCV 
preperation, Sepharose CL-4B gel-filtration chromatography, phosphocellulose cation exchange chromatography, Superose 6 FPLC and observed that the CALM protein was able to assemble the clathrin into clathrin cage.

\section{Materials and Methods}

\section{Materials}

All buffers used in the protein study, even though it is not explicitly indicated in the citation, contained $0.1 \mathrm{mM}$ PMSF. All chemicals were purchased from USB and Sigma. Centricon-30 was from Amicon. NBT/BCIP reagents were from Boehringer Mannheim. All chromatography resins and PVDF membrane were from Amesham Pharmacia. Monoclonal antibody against CALM was prepared by immunizing the bacterially expressed GST-CALM protein (Kim et al., 2000). Monoclonal antibody against AP180 was kindly provided by Dr. S.R. Kim.

\section{Preperation of clathrin-coated vesicles}

Rat liver was minced and homogenized with six passes of Potter-Elvejhem tissue grinder in an equal volume of ice-cold homogenating buffer to the weight of the liver. The homogenizing buffer consisted of $0.1 \mathrm{M} 2-(\mathrm{N}-$ morpholino) ethanesulfonic acid (MES), $1 \mathrm{mM}$ EGTA, $0.5 \mathrm{mM} \mathrm{MgCl}_{2}$, and $0.02 \% \mathrm{NaN}_{3}$ adjusted to $\mathrm{pH} 6.5$ with sodium hydroxide. The homogenate was centrifuged at $19,000 \times g$ in a Sorval GSA rotor for $40 \mathrm{~min}$. The supernatant was saved and centrifuged for $70 \mathrm{~min}$ at 43,000 $\mathrm{x} g$ in a Sorvall SS34 roter. The pellet containing the coated vesicles was resuspended in a small volume of homogenizing buffer using a loose-fitting Dounce homogenizer. The resulting pellet is crude coated-vesicle.

\section{Extraction of coating proteins}

The coated vesicles were extracted according to the procedure of Keen et al.. The crude coated-vesicle was resuspended in the extraction buffer, which composed of $0.5 \mathrm{M}$ Tris, $0.1 \mathrm{mM}$ phenylmethylsulfonyl fluoride (PMSF), pH 7.0, 2 mM dithiothreitol (DTT), 1 mM EDTA, and homogenized using Dounce glass homogenizer. The homogenate was stored at 4 overnight, and clarified by ultracentrifugation at $100,000 \times g$ for $3 \mathrm{~h}$.

\section{Gel-filtration chromatography}

The tris buffer extract was applied to the Sepharose CL4B (Pharmacia, Upsala, Sweden) column $(2 \times 100 \mathrm{~cm})$ equilibrated with the extraction buffer and eluted with the equilibration buffer with the flow rate of $0.5 \mathrm{ml} / \mathrm{min}$. Aliquots of all fractions were analyzed by SDS-PAGE and immuno-blotted with CALM monoclonal antibody.
Fractions that contained partially separated assembly proteins were pooled and concentrated by Centricon 30 .

\section{Phosphocellulose chromatography}

For a further purification, fractions of the assembly protein were chromatographed on phosphocellulose column $(1.0 \times 10 \mathrm{~cm})$ using FPLC system. The sample was applied to the column equilibrated with $50 \mathrm{mM}$ potassium phosphate buffer, $\mathrm{pH} 6.0$. and washed with $10 \mathrm{ml}$ of equilibration buffer, and then, eluted with a $30 \mathrm{ml}$ gradient consisting of $1 \mathrm{mM}$ sodium phosphate, $1 \mathrm{mM}$ DTT, $\mathrm{pH} 8.8$ and $350 \mathrm{mM}$ potassium phosphate in $1 \mathrm{mM}$ DTT, $\mathrm{pH}$ 8.8. Fractions of $0.5 \mathrm{ml}$ were collected.

\section{Superose 6 Gel filtration fast performance Liquid chromatography (FPLC)}

The sample from the gel-filtration chromatography was applied to the Superose 6 column equilibrated with the extraction buffer. Then the column was eluted with equilibration buffer.

\section{Preperation of clathrin}

Rat clathrin was used for the binding of clathrin assembly proteins and the clathrin-cage formation. The extraction of coating proteins and the gel-filtration procedures were same as above. The first protein peak was applied to the Superose 6 FPLC, and the major protein peak was used for the clathrin-assembly study.

\section{Clathrin assembly assay}

Three micro molar clathrin triskelia were dialyzed overnight at $4^{\circ} \mathrm{C}$ against isolation buffer (0.1 M MES, $\mathrm{pH} 6.7$, containing $1 \mathrm{mM}$ EGTA, $0.5 \mathrm{mM} \mathrm{MgCl}$, and $0.1 \mathrm{mM}$ PMSF) with an addiation of $20 \mu \mathrm{M}$ assembly protein (purified CALM protein and GST). Following the $3 \mathrm{~min}$ centrifugation at $13,600 \times g$ to remove nonspecific aggregates, newly assembled clathrin cages were collected by ultracentrifugation for $20 \mathrm{~min}$ at $100,000 \times \mathrm{g}$. Fractions of the pellet $(P)$ and supernatant $(S)$ were analyzed by SDS-PAGE, followed by Coomassie blue staining.

\section{Results and Discussion}

\section{Purification of assembly proteins}

Clathrin-coated vesicles were isolated from rat liver by the procedure described by Ahle and Ungewickell (1986). Clathrin and its associated membrane proteins were liberated from the purified coated vesicles with $0.5 \mathrm{M}$ Tris at $\mathrm{pH} 7.0$ and the membrane fractions were removed by ultracentrifugation. The supernatant contained most of the clathrin and other clathrin associated proteins. In order to isolate clathrin, molecular sieve on a Sepharose CL-4B column equilibrated with the extraction buffer to 
A

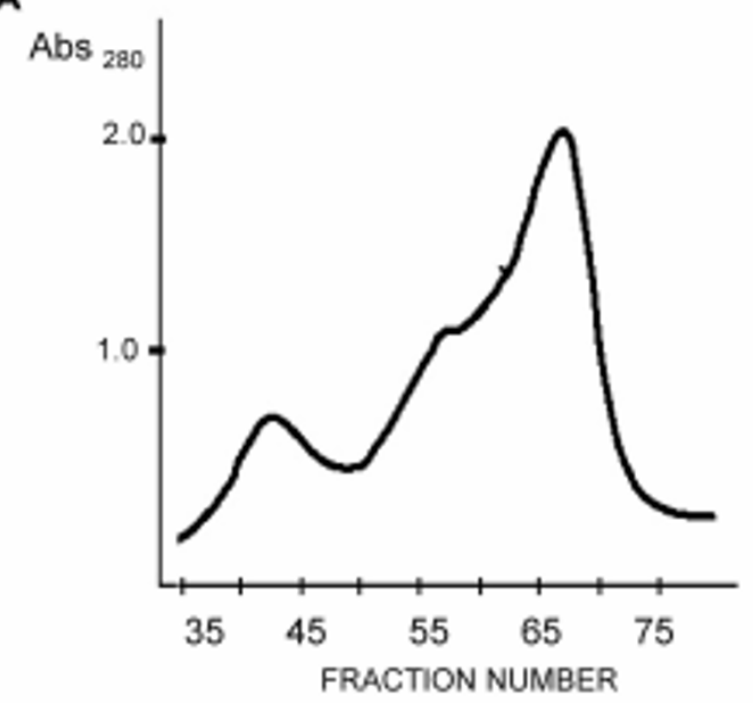

B

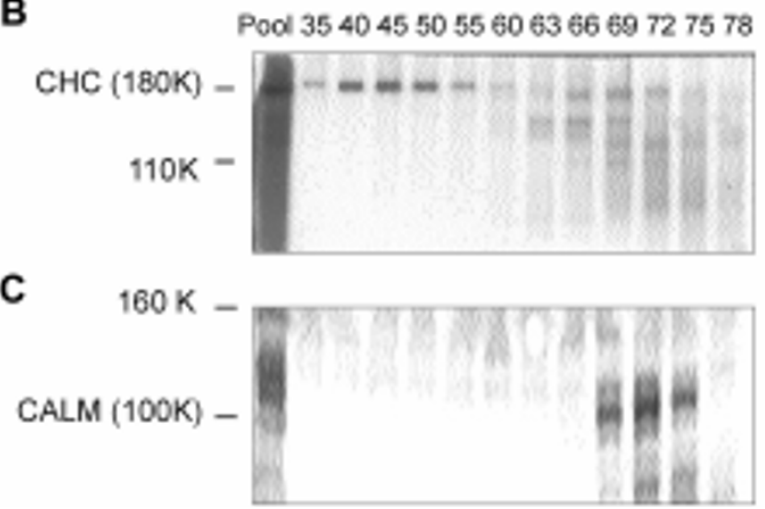

Figure 1. Gel filration of solubilized coat protein, electrophoresis and immunoblot analysis of individual Sepharose $\mathrm{CL}-4 \mathrm{~B}$ column fractions. (A) The coated vesicles were incubated with $0.5 \mathrm{M}$ Tris- $\mathrm{HCl}, 2 \mathrm{mM}$ EDTA, $1 \mathrm{mM}$ $\mathrm{DTT}, \mathrm{pH}$ 7.0. The extracted proteins were clarified with ultracentrifugation and then subjected to gel-filtration on Sepharose CL-4B column. (B) Aliquots of fractions of gel filtration elution were analysed by SDS-PAGE and the separated proteins were either stained for protein by Coomassie blue (B) or electro-blotted on to PVDF membrane and reacted with monoclonal antibody of CALM (C)

maintain the assembly proteins (i.e. AP-1, $-2,-3$, etc.) in a dissociated state. On the gel filtration profile from the liver tissue, the first peak is the most prominent (Prasad and Lippoldt, 1988). Figure 1 shows a typical elution profile from liver tissue where the first peak is moderate. Analysis of the column fractions by SDS-PAGE shows that the first peak contains clathrin triskelia. The first peak from the liver extract was a bit smaller, which reflect that vesicle trafficking may be lower in liver tissue than in brain. The Western blot analysis of the eluents revealed that the first peak was not immunoreactive against recombinant CALM, but the latter part of the elutes have two immunoreactive bands. The faint band has molecular weight of $100 \mathrm{kD}$, and the lower intense band was about $90 \mathrm{kD}$. The two bands might be
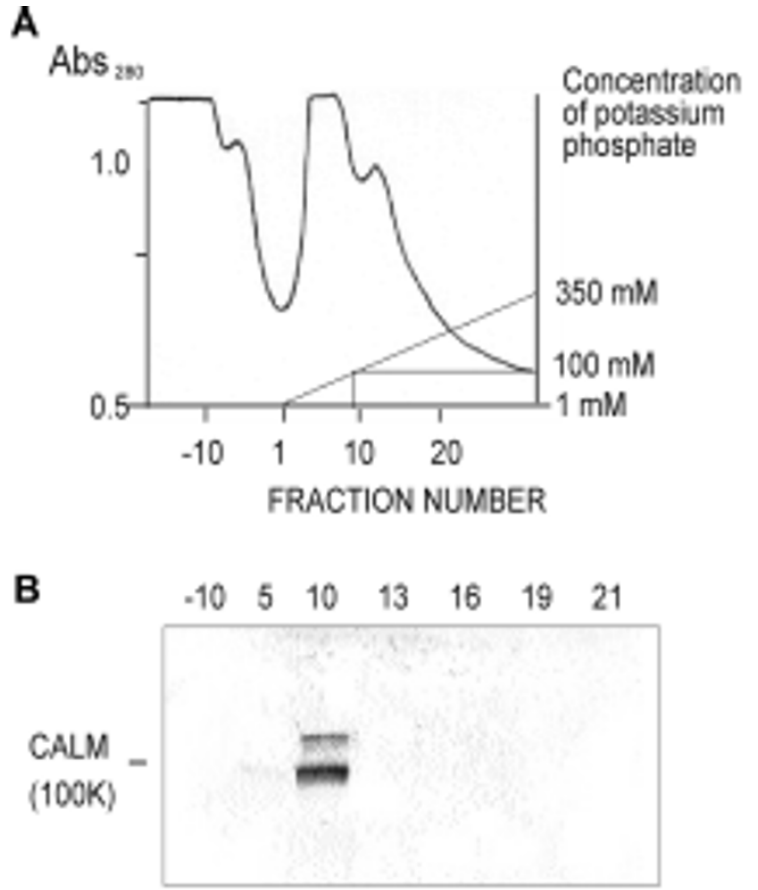

Figure 2. Chromatogram of CALM proteins on phosphocellulose cationexchange chromatography. The third peak of immunoreaction for CALM antibody from the Sepharose CL-4B gel-filtration chromatography was applied to phosphocellulose cation exchange column. The column was eluted with a linear 1-0.35 M potassium phosphate gradient (see 'Materials and methods' for details). Fractions containing protein were analysed by Western blot.

ascribed to the alternatively splicing variants of the gene (Kim and Lee, 1999).

The size of these bands was somewhat larger than that of bacterial expression product of CALM, $80 \mathrm{kD}$ (Kim et al. 2000). On translation of the CALM gene, this protein is composed of 640 amino acids with estimated molecular weight of $71 \mathrm{kD}$. The increase in apparent molecular weight would be resulted from the atypical amino acid composition and postranslational modification. The protein contained highly positively charged amino acids and proline residues (45 proline/640 residues), that caused anormalous retarded migration of the CALM on SDS-PAGE, and resulted in the differences between true and apparent molecular weights. In mammals, furthermore, the increase in apparent molecular weight might be caused by posttranslational glycosylation or phosphorylation modifications of the primary translation product. For these reasons, the $100 \mathrm{kD}$-sized proteins, which is immunoreactive against anti-CALM antibody, were considered to the native form and purified throughout the procedure.

The estimated pl value of the CALM trnascript is 8.7 and that of AP180 is 4.5 (Kim and Lee, 1999). In contrast to using anion exchange chromatography for purification of AP180 due to its acidic nature (Ahle and Ungewickell, 1986), we chose cation exchange chromato- 


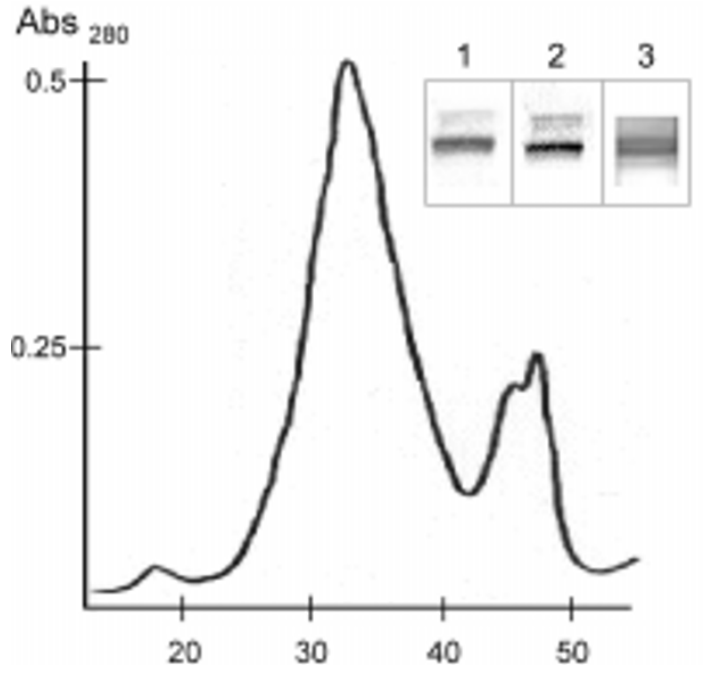

Figure 3. Superose 6 FPLC elution profile of pooled fraction from phosphocellulose cation-exchange chromatography. The pooled fraction was concentrated with Centricon 30 and applied to Superose 6 FPLC column. The column was eluted with $0.5 \mathrm{M}$ Tris, $\mathrm{pH} 7.5$, and the proteins were resolved into two major peaks. The first peak was pooled, separated on SDS-PAGE, and detected with Coomassie blue protein staining (1), antiCALM antibody (2), and anti AP180 antibody (3).

graphy for the next purification step. The fractions containing the $100 \mathrm{kD}$ protein were pooled and subjected to chromatography on a phosphocellulose cation-exchange column in conjunction with FPLC system. The column was equilibrated with $50 \mathrm{mM}$ potassium phosphate buffer, $\mathrm{pH}$ 6.0. The bound sample was eluted with linear gradient of $350 \mathrm{mM}$ potassium phosphate buffer, $\mathrm{pH}$ 8.8. As shown on Figure 2 more than two third of the protein was in the wash fraction and the 90-100 kD protein was eluted at the front part of phosphate gradient, about $100 \mathrm{mM}$ potassium phosphate concentration.

The protein with molecular weight of $90-100 \mathrm{kD}$ was further purified by Superose 6 FPLC. The pooled fraction was concentrated with Centricon-30 and applied to Superose 6 column. The sample was resolved into two major peaks, and the first peak was immuroreactive to anti-CALM antibody (Figure 3). On SDS-PAGE analysis of the pooled sample, the $90 \mathrm{kD}$-sized band was prominent whereas the $100 \mathrm{kD}$ sized band was faint. The monoclonal antibody might be more reactive to the short variant of the alternatively spliced CALM.

There was a remarkable homology between the primary structure of the CALM and AP180. The homology of more than $95 \%$ is seen between the first 289 amino acids of CALM and those of AP180 (Kim and Lee, 1999). Western blot analysis was carried out for the purified CALM using monoclonal antibody against AP180. The proteins were also reactive to AP180 antibody (Figure 3, lane 3), suggesting that the tertiary structure of $\mathrm{N}$-terminal region of AP180 and CALM would be similar. Furthermore, the $\mathrm{N}$-terminal region of the AP180

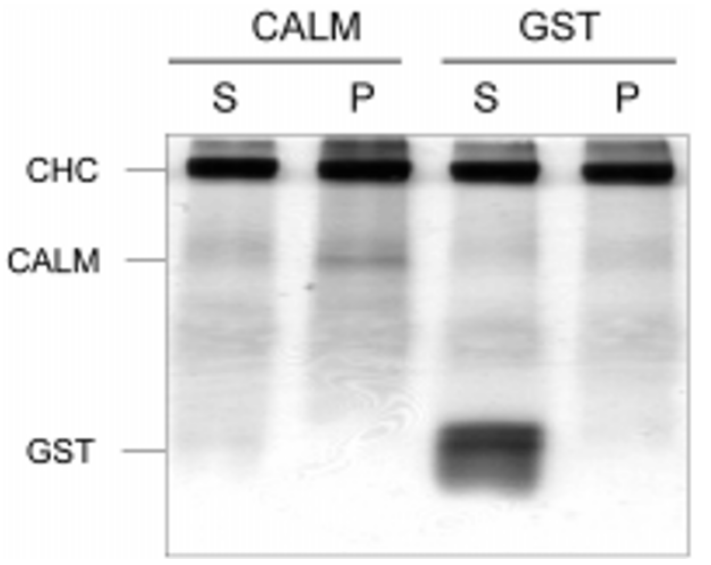

Figure 4. Assembly of Clathrin cage by the CALM protein. Clathrin assembly assays were performed as described on 'Materials and Methods'. $3 \mu \mathrm{M}$ clathrin triskelia was dialyzed overnight at $4^{\circ} \mathrm{C}$ against isolation buffer with the addition of $20 \mu \mathrm{M}$ purified CALM (lane 1 and 2), GST (lane 3 and 4). Following a low speed centrifugation to remove nonspecific aggregates, newly assembled clathrin cages were pelleted by ultracentrifugation at $100,000 \mathrm{~g}$. The supernatant $(\mathrm{S})$ and the pellet $(\mathrm{P})$ fractions were analyzed by $10 \%$ SDS-PAGE, followed by Coomassie blue staining. Positions of clathrin heavy chain (CHC), CALM and GST were indicated.

where the binding site for the inositol and inositides (Hao et al., 1997) is present, may compete with the CALM for the inositides and inositol phosphates, the regulators of clathrin cage formation, and would play a crucial role in membrane trafficking.

\section{Clathrin assembly assay}

CALM is homologous protein of AP180. Apparently, both $\mathrm{N}$ terminal $(33 \mathrm{kD}$ ) and $\mathrm{C}$ terminal $(55 \mathrm{kD})$ parts of the AP180 have clathrin binding activity (Ye and Lafer, 1995) and C terminal domain has the activity of clathrin cage assembly. Recently it was reported that the CALM could bind to clathrin heavy chain (Teber et al., 1999). Moreover, CALM of GST fusion protein could bind to preassembled clathrin cage and could assemble clathrin cage (Kim et al., 2000). Clathrin triskelia was dialyzed with putative assembly protein in isolation buffer to induce clathrin assemble into cages. When CALM was mixed with the clathrin triskelia, bound CALM cosedimented with the cages at $100,000 \times \mathrm{g}$, while free CALM remained in the supernatant. When clathrin triskelia was sedimented following a dialysis in the presence of purified CALM, it remained in the pellet with the clathrin cages, but a negative control GST was not cosedimented with clathrin cages (Figure 4). These results indicated that the purified CALM has an ability to assemble the clathrin cage.

\section{Acknowledgement}

This study was supported by the Grant awarded to 
Hyung-Lae Kim from the Ewha Womans University, in 1998.

\section{References}

Ahle, S. and Ungewickell E. (1986) Purification and properties of a new clathrin assembly protein. EMBO J. 5: 3143-3149

Dreyling, M. H., Martinez-Climent, J. A., Zheng, M., Mao, J., Rowley, J. D. and Bohlander. S. K. (1996) The t(10;11)(p13; q14) in the U937 cell line results in the fusion of the AF10 gene and CALM, encoding a new memberr of the AP-3 clathrin assembly protein family. Proc. Natl. Aca. Sci. USA 93: 4804-4809

Hao, W., Tan, Z., Prasad, K., Reddy, K., Che, J., Prestwich, G. D., Falck, J. R., Shears, S. B. and Lafer, E. M. (1997) Regulation of AP-3 function by inositides: lentification of phosphatidyl inositol 3,4,5-tripphosphate as a potent ligand. J. Biol. Chem. 272: 6393-6398

Kim, J.-A., Kim, S.-R., Jung, Y.-K., Woo, S.-Y., Seoh, J.-Y., Hong, Y.-S. and Kim, H.-L. (2000) Properties of GST-CALM expressed in E. coli. Exp. Mol. Med. 32: 93-99

Kim, H.-L. and Lee, S. C. (1999) Molecular cloning of clathrin assembly protein gene (rCALM) and its differential expression to AP180 in rat brain. Exp. Mol. Med. 31: 191-196

Kondury, P. and Roland, E. L. (1988) Molecular characterization of the AP180 coated vesicle assembly protein. Biochem. 27: 6098-6104

Morris, S. A., Schroder, S., Plessmann, U., Weber, K. and Ungewickell, E. (1993) Clathrin assembly protein AP 180: primary structure, domain organization and identification of a clathrin binding site. EMBO J. 12: 667-675

Norris, F. A., Ungewickell, E. and Majerus, P. W. (1995) Inositol hexakisphosphate binds to clathrin assembly protein 3(AP3/AP180) and inhibits clathrin cage assembly in vitro. J. Biol.

\section{Chem. 270: 214-217}

Prasad, K. and Lippoldt, R. E. (1988) Molecular characterization of AP180 coated vesicle assembly protein. Biochem. 27: 6908-6104

Pearse, B. M. F and Robinson, M. S. (1990) Clathrin, adaptors, and sorting. Ann. Rev. Cell Biol. 6: 151-171

Robinson, M. S. (1994) The role of clathrin, adaptors, and dynamin in endocytosis. Curr. Opin. Cell Biol. 6: 538-544

Stephen, A. M., Annette, M. and Ungewickell (1990) Anaysis of 100-180 kD phosphoprotein in clathrin-coated vesicles from bovine brain. J. Biol. Chem. 265: 3354-3357

Tebar, F., Bohlander, S. K. and Sorkin, A. (1999) Clathrin assembly lymphoid myeloid leukemia (CALM) protein: localization in endocytic-coated pits, interaction with clathrin, and the impact of overexpression on clathrin-mediated traffic. Mol. Biol. Cell 10: 2687-2702

Wilde, A. and Brodsky, F. M. (1996) In vivo phosphorylation of adaptors regulaates their interaction with clathrin. J. Cell Biol. 135: 635-645

Ye, W. and Lafer, E. M. (1995) Clathrin binding and assembly activities of expressed domains of the synapse-specific clathrin assembly protein AP-3. J. Biol. Chem. 270: 10933-10939

Ye, W., Ali, N., Bembenek, M.,E., Shears, S. B. and Lafer, E. M. (1995) Inhibition of clathrin assembly by high affinity binding of specific inositol polyphosphate to the synapse-specific clathrin assembly protein AP-3. J. Biol. Chem. 270: 15641568

Zhou, S., Sousa, R. and Lafer, E. M.(1992) Characterization of a novel synapse-specific protein II. cDNA cloning and sequence analysis of F1-20 protein. J. Neurochem. 12: 21142155

Zhou, S., Tannery, N. and H, Lafer, E. M. (1993) The synapsespecific phosphoprotein $\mathrm{F} 1-20$ is identical to the clathrin assembly protein AP-3. J. Biol. Chem. 268: 12655-12662 